\title{
A Table of Integrals of the Error Function. II. Additions and Corrections*
}

\author{
Murray Geller** and Edward W. Ng**
}

(September 28, 1971)

\begin{abstract}
This is an extension of a compendium of indefinite and definite integrals of products of the error function with elementary or transcendental functions recently published by the authors.

Key words: Error functions; indefinite integrals; special functions.
\end{abstract}

\section{Introduction}

Since the present authors published an extensive compendium of integrals involving the error function $[1],{ }^{1}$ numerous comments and suggestions have been received. In particular, we have been advised by Р. І. Нadji (П. И. ХАДЖИ) of his publication in Russian [2], which contains formulas not included in [1]. Careful examination of [2] has revealed numerous mathematical and typographical errors. Bearıng in mind also the inaccessibility of his report, the authors believe it to be appropriate to publish an extension of [1] based largely on corrected versions of Hadji's formulas but also containing new results. It should be noted that the authors have verified and/or corrected all of Hadji's formulas in addition to rederiving and checking all of the formulas in [1].

Throughout this article, we conform to the format and notation of [1], including section and equation numbers. A supplementary glossary is also included. Within each subsection, typographical errors, equivalent forms and errors detected in [1] will be listed under corrections.

\section{Supplementary Glossary}

$\begin{array}{lll}B(p, q) & \text { Beta Function } & \Gamma(p) \Gamma(q) / \Gamma(p+q) \\ \beta(x) & & \frac{1}{2}\left[\psi\left(\frac{1}{2}+\frac{1}{2} x\right)-\psi\left(\frac{1}{2} x\right)\right] \\ C_{n}^{\lambda}(x) & \text { Gegenbauer Polynomial } & \\ & & \\ G & \text { Catalan's Constant } & 0.915965594 \ldots\end{array}$

$P_{n}^{(\alpha, \beta)}(x) \quad$ Jacobi Polynomial

$T_{n}(x) \quad$ Chebyshev Polynomial

AMS Subject Classification: Primary 6505

* An invited paper. This paper presents the results of one phase of research carried out at the Jet Propulsion Laboratory, California Institute of Technology, under Contract \#NAS7-100, sponsored by the National Aeronautics and Space Administration.

** Present address: Jet Propulsion Laboratory, California Institute of Technology, Pasadena, California 91103.

${ }^{1}$ Figures in brackets indicate the literature references at the end of this paper. 


\section{Integrals of Products of Error Functions with Other Functions}

\subsection{Combination of Error Function with Powers}

Additions

(20)

$$
\int_{a}^{\infty} \operatorname{erf}(x) x^{-p} d x=\frac{1}{p-1}\left[\frac{\operatorname{erf}(a)}{a^{p-1}}+\frac{1}{\sqrt{\pi}} \frac{e^{-a^{2 / 2}}}{a^{p / 2}} W_{-p / 4,(p-2) / 4}\left(a^{2}\right)\right], \quad p>1
$$$$
\int_{0}^{\infty}[\operatorname{erf}(a x+c)-\operatorname{erf}(b x+c)] \frac{d x}{x}=\ln \left(\frac{a}{b}\right) \operatorname{erfc}(c)
$$

$\int_{0}^{\infty}[b \operatorname{erf}(a x)-a \operatorname{erf}(b x)] \frac{d x}{x^{2}}=\frac{2 a b}{\sqrt{\pi}} \ln \left(\frac{b}{a}\right)$

$\int_{0}^{\infty}[\sqrt{x} \operatorname{erf}(\sqrt{x})-\operatorname{erf}(x)] \frac{d x}{x^{2}}=\frac{1}{\sqrt{\pi}}(2-\gamma)$

$\int_{0}^{\infty}\left[\frac{\operatorname{erf}(x)}{x}-\frac{2}{\sqrt{\pi}} \frac{1}{1+x^{2}}\right] \frac{d x}{x}=\frac{1}{\sqrt{\pi}}(2-\gamma)$

(25) $\int_{0}^{u}(u-x)^{p} \operatorname{erf}[\sqrt{(a / x)}] d x=\frac{u^{p+1}}{p+1}\left[1-\frac{\Gamma(p+2)}{\sqrt{ } \pi}\left(\frac{u}{a}\right)^{1 / 4} e^{-a /(2 u)} W_{-p-(5 / 4), 1 / 4}\left(\frac{a}{u}\right)\right], \quad p>-1$

(26) $\int_{0}^{\infty}\left[(b+i x)^{-p-1}-(b-i x)^{-p-1}\right] \operatorname{erf}(a x) d x=-\frac{2 i}{p}(a \sqrt{2})^{p} e^{a^{2} b^{2} / 2} D_{-p}(a b \sqrt{2}), \quad p>0$

(27) $\int_{0}^{\infty} x\left[(b+i x)^{-p-1}+(b-i x)^{-p-1}\right] \operatorname{erf}(a x) d x$

$$
=\frac{2}{p(p-1)}(\alpha / b)^{p-1} e^{a^{2} b^{2} / 2}\left[(p-1) \alpha D_{-p}(\alpha)-p D_{1-p}(\alpha)\right], \quad p>1, \alpha=a b \sqrt{2}
$$

(31) $\int_{0}^{u} x^{2 \nu-1}\left(u^{2}-x^{2}\right)^{\mu-1} \operatorname{erf}(a x) d x$

$$
\begin{gathered}
=\frac{a}{\sqrt{\pi}} u^{2 \mu+2 \nu-1} B\left(\mu, \nu+\frac{1}{2}\right)_{2} F_{2}\left(\frac{1}{2}, \nu+\frac{1}{2} ; \frac{3}{2}, \mu+\nu+\frac{1}{2} ;-a^{2} u^{2}\right), \mu>0, \nu>-\frac{1}{2} \\
\int_{0}^{\infty} \frac{x \operatorname{erf}(a x)}{\left(x^{2}+b^{2}\right)^{2}} d x=\frac{a}{2 b} \sqrt{\pi} e^{a^{2} b^{2}} \operatorname{erf} c(a b)
\end{gathered}
$$

*Section 3 corresponds to section 4 of reference [1]. 


$$
\int_{0}^{1} \frac{x \operatorname{erf}(a x)}{\left(x^{2}+1\right)^{2}} d x=\frac{a}{4} \sqrt{\pi} e^{a^{2}}\left[1-\operatorname{erf}^{2}(a)\right]-\frac{1}{4} \operatorname{erf}(a)
$$

$$
\int_{1}^{\infty} \frac{x \operatorname{erf}(a x)}{\left(x^{2}+1\right)^{2}} d x=\frac{a}{4} \sqrt{\pi} e^{a^{2}}[1-\operatorname{erf}(a)]^{2}+\frac{1}{4} \operatorname{erf}(a)
$$

$\int_{0}^{\infty} \frac{x \operatorname{erf}(a x)}{\left(x^{2}+b^{2}\right)^{p+1}} d x=\frac{a}{2} b^{1-2 p} \frac{\Gamma\left(p-\frac{1}{2}\right)}{\Gamma(p+1)}{ }_{1} F_{1}\left(\frac{1}{2} ; \frac{3}{2}-p ; a^{2} b^{2}\right)$

$$
+\frac{a^{2 p}}{2 \sqrt{\pi}} \frac{\Gamma\left(\frac{1}{2}-p\right)}{\Gamma(p+1)}{ }_{1} F_{1}\left(p ; p+\frac{1}{2} ; a^{2} b^{2}\right), p>0, p \neq n+\frac{1}{2}
$$

$$
\begin{gathered}
\int_{0}^{\infty} \frac{\operatorname{erf}(a x)}{\left(x^{2}+b^{2}\right)^{3 / 2}} d x=\frac{1}{b^{2}}\left[1-\exp \left(a^{2} b^{2}\right) \operatorname{erf} c(a b)\right] \\
\int_{0}^{\infty} \frac{x \operatorname{erf}(a x)}{\left(x^{2}+b^{2}\right)^{3 / 2}} d x=\frac{a}{\sqrt{\pi}} \exp \left(a^{2} b^{2} / 2\right) K_{0}\left(\frac{a^{2} b^{2}}{2}\right) \\
\int_{0}^{\infty} \frac{x \operatorname{erf}\left[a \sqrt{\left.\left(x^{2}+b^{2}\right)\right]}\right.}{\left(x^{2}+b^{2}\right)^{3 / 2}} d x=\frac{1}{b} \operatorname{erf}(a b)+\frac{a}{\sqrt{\pi}} E_{1}\left(a^{2} b^{2}\right) \\
\int_{0}^{\infty} \frac{x \operatorname{erf}[\sqrt{(a x)}]}{\left(x^{2}+4 b^{2}\right)^{3 / 2}} d x=\frac{a \pi}{2 \sqrt{2}}\left[J_{1 / 4}(a b) Y_{-1 / 4}(a b)-J_{-1 / 4}(a b) Y_{1 / 4}(a b)\right]
\end{gathered}
$$

\subsection{Combination of Error Function with Exponentials and Powers}

\section{Corrections}

(10) Replace $\left(b z^{n}+n z^{n-1}\right)$ in last expression by $\left(b z^{n-1}+(n-1) z^{n-2}\right)$.

(12) Replace $\left(b z^{n}+n z^{n-1}\right)$ in last expression by $\frac{1}{a \sqrt{\pi}}\left(b z^{n-1}+(n-1) z^{n-2}\right)$.

(13) Replace $(2 a)^{(k-n) / 2}$ by $\left(2 a^{2}\right)^{(k-n) / 2}$.

\section{Additions}

$$
\int_{0}^{\infty}\left[\operatorname{erf}\left(e^{-a x}\right)-\operatorname{erf}\left(e^{-b x}\right)\right] \frac{d x}{x}=\operatorname{erf}(1) \ln \left(\frac{b}{a}\right)
$$

$$
\int_{-\infty}^{\infty} \operatorname{erf}\left[\frac{a e^{-i p x}}{(b \pm i x)^{\alpha}}\right] \frac{d x}{x^{2}+c^{2}}=\frac{\pi}{c} \operatorname{erf}\left[\frac{a e^{\bar{p} p}}{(b \pm c)^{\alpha}}\right], p>0, \alpha \geqslant 0, b \neq c \text { for the lower sign }
$$

$$
\int_{-\infty}^{\infty} \operatorname{erf}\left(\frac{a}{b \pm i x}\right) e^{-i p x} \frac{d x}{x^{2}+c^{2}}=\frac{\pi}{c} e^{-p c} \operatorname{erf}\left(\frac{a}{b \pm c}\right), p>0, b \neq c \text { for the lower sign }
$$

$$
\begin{gathered}
\int_{-\infty}^{\infty}(i x)^{-p} \operatorname{erf}\left(a e^{-i b x}\right) \frac{d x}{x^{2}+c^{2}}=\frac{\pi}{c^{p+1}} \operatorname{erf}\left(a e^{-b c}\right),|p|<1 \\
f_{-\infty}^{\infty} \operatorname{erf}\left(a e^{ \pm i b x}\right) \frac{d x}{x-c}= \pm i \pi \operatorname{erf}\left(a e^{ \pm i b c}\right)
\end{gathered}
$$




$$
\int_{0}^{\infty} \frac{d x}{\sin h \frac{1}{2} \pi x}\left[\frac{\operatorname{erf}(1+i x)}{(1+i x)^{2}}-\frac{\operatorname{erf}(1-i x)}{(1-i x)^{2}}\right]=2 i \operatorname{erf}(1)
$$

\subsection{Combination of Error Function with Exponentials of More Complicated Arguments}

Corrections

(2) Equivalent form: $\int_{0}^{\infty} \operatorname{erf}(a x) e^{-b^{2} x^{2}} d x=\frac{1}{b \sqrt{\pi}} \tan ^{-1}\left(\frac{a}{b}\right)$

(3) Remove condition " $b$ may be complex" and replace by $\mathscr{R}\left(a^{2}\right)>\mathscr{R}\left(b^{2}\right)$

(8) For the form given, add the condition $\mathscr{R}\left(b^{2}\right)>\mathscr{R}\left(a^{2}\right)$

Equivalent form:

$$
\begin{gathered}
\int_{0}^{\infty} \operatorname{erf}(a x) e^{-b^{2} x^{2}} x^{p} d x=\frac{a}{b^{p+1} \sqrt{\pi}} \frac{\Gamma\left(1+\frac{1}{2} p\right)}{\left(a^{2}+b^{2}\right)^{\frac{1}{2}}}{ }_{2} F_{1}\left(\frac{1}{2}-\frac{1}{2} p, \frac{1}{2} ; \frac{3}{2} ; \frac{a^{2}}{a^{2}+b^{2}}\right), \\
\mathscr{R}\left(a^{2}\right)>0, \mathscr{R}\left(b^{2}\right)>0, p>-2 .
\end{gathered}
$$

$$
\int_{1}^{\infty} \operatorname{erf} c(a x) e^{a^{2} x} x^{-3} d x=\frac{1}{2}\left(1+2 a^{2}\right) e^{a^{2}} \operatorname{erf} c(a)-a \pi^{-1 / 2}
$$

(35) Add the condition $\mathscr{R}\left(c^{2}\right)>\mathscr{R}\left(a^{2}\right)$

(36) Replace $K_{b}\left(a^{2}\right)$ by $K_{b}\left(\frac{1}{2} a^{2}\right)$

\section{Additions}

$$
\int x \exp \left(x^{2}\right) \operatorname{erf}(x) d x=\frac{1}{2} \exp \left(x^{2}\right) \operatorname{erf}(x)-\frac{1}{\sqrt{\pi}} x
$$

$$
\int_{-a}^{a} \exp \left(x^{2}\right) \operatorname{erf} c(x) d x=\sqrt{\pi} \operatorname{erf} i(a)
$$

$$
\int[\operatorname{erf}(a x)]^{n} \exp \left(-a^{2} x^{2}\right) d x=\frac{\sqrt{ } \pi}{2 a(n+1)}[\operatorname{erf}(a x)]^{n+1}
$$

$$
\int\left[a \operatorname{erf}(b x) \exp \left(-a^{2} x^{2}\right)+b \operatorname{erf}(a x) \exp \left(-b^{2} x^{2}\right)\right] d x=\frac{\sqrt{\pi}}{2} \operatorname{erf}(a x) \operatorname{erf}(b x)
$$

(43) $2 b^{2} \int \operatorname{erf}(a x+c) \exp \left(-b^{2} x^{2}\right) x d x$

$$
\begin{gathered}
=\frac{a}{\sqrt{\left(a^{2}+b^{2}\right)}} \operatorname{erf}\left(x \sqrt{\left(a^{2}+b^{2}\right)}+\frac{a c}{\sqrt{\left(a^{2}+b^{2}\right)}}\right) \exp \left(-\frac{b^{2} c^{2}}{a^{2}+b^{2}}\right)-\operatorname{erf}(a x+c) \exp \left(-b^{2} x^{2}\right) \\
\int x^{2 n} \operatorname{erf}(a x) \exp \left(-a^{2} x^{2}\right) d x=\frac{(2 n) !}{n !} \frac{\sqrt{\pi}}{(2 a)^{2 n+1}} A,
\end{gathered}
$$


where

$A=\frac{1}{2} \operatorname{erf}^{2}(a x)-\frac{2 a}{\sqrt{\pi}} x \operatorname{erf}(a x) \exp \left(-a^{2} x^{2}\right) \sum_{k=0}^{n-1} \frac{k !}{(2 k+1) !}\left(4 a^{2} x^{2}\right)^{k}$

$$
-\frac{\exp \left(-2 a^{2} x^{2}\right)}{\pi} \sum_{k=0}^{n-1} 2^{k} \frac{k ! k !}{(2 k+1) !} e_{k}\left(2 a^{2} x^{2}\right)
$$

$$
\int x^{2 n+1} \operatorname{erf}(a x) \exp \left(-a^{2} x^{2}\right) d x=\frac{n !}{a^{2 n+2}} B
$$

- where

$B=2^{-3 / 2} \operatorname{erf}(a x \sqrt{2}) \sum_{k=0}^{n}\left(\begin{array}{c}2 k \\ k\end{array}\right) 8^{-k}-\frac{1}{2} \operatorname{erf}(a x) e^{-a^{2} x^{2}} e_{n}\left(a^{2} x^{2}\right)$

$$
-\frac{a x}{\sqrt{\pi}} e^{-2 a^{2} x^{2}} \sum_{k=1}^{n}(\underset{k}{2 k}) 8^{-k} \sum_{l=0}^{k-1} \frac{l !}{(2 l+1) !}\left(8 a^{2} x^{2}\right)^{l}
$$

(46) $2 b^{2} \int x^{2 n+1} \operatorname{erf}(a x) \exp \left(-b^{2} x^{2}\right) d x=2 n \int x^{2 n-1} \operatorname{erf}(a x) \exp \left(-b^{2} x^{2}\right) d x$

$$
-x^{2 n} \operatorname{erf}(a x) \exp \left(-b^{2} x^{2}\right)+\frac{2 a}{\sqrt{\pi}} \int x^{2 n} \exp \left[-\left(a^{2}+b^{2}\right) x^{2}\right] d x
$$

(47) $\int \operatorname{erf}(a x) \exp \left(-a^{2} x^{2}\right) \frac{d x}{x^{2}}=-\frac{1}{x} \operatorname{erf}(a x) \exp \left(-a^{2} x^{2}\right)-\frac{a \sqrt{\pi}}{2} \operatorname{erf}^{2}(a x)-\frac{a}{\sqrt{\pi}} E_{1}\left(2 a^{2} x^{2}\right)$

(48) $\int \exp \left\{-b^{2}[\operatorname{erf}(a x)]^{p}\right\} \exp \left(-a^{2} x^{2}\right)[\operatorname{erf}(a x)]^{q} d x$

$$
=\frac{\sqrt{\pi}}{2 a p} b^{-2(1+q) / p} \gamma\left(\frac{q+1}{p}, b^{2}[\operatorname{erf}(a x)]^{p}\right), q>-1, p>0
$$

(49)

$$
\int \frac{e^{-a^{2} x^{2}}}{\operatorname{erf}(a x)} d x=\frac{\sqrt{\pi}}{2 a} \ln [\operatorname{erf}(a x)]
$$

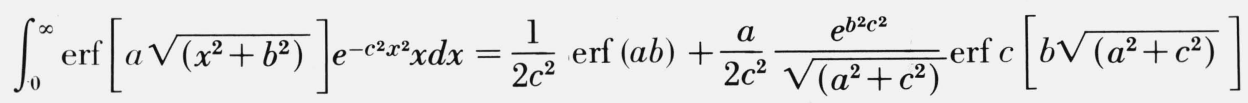

$$
\begin{gathered}
\int_{0}^{\infty} \operatorname{erf}[\sqrt{(a x)}] e^{-b / x} \frac{d x}{x^{2}}=\frac{1}{b}\left[1-e^{-2 \sqrt{(a b)}}\right] \\
\int_{-\infty}^{\infty} \operatorname{erf}\left(a e^{x / 2}\right) e^{x / 2} \exp \left(-e^{x}\right) d x=\frac{2}{\sqrt{\pi}} \tan ^{-1} a \\
\int_{0}^{\infty}\left[\frac{a \operatorname{erf}\left(\alpha \exp \left[-c e^{a x}\right]\right)}{1-e^{-a x}}-\frac{b \operatorname{erf}\left(\alpha \exp \left[-c e^{b x}\right]\right)}{1-e^{b x}}\right] d x=\ln \left(\frac{b}{a}\right) \operatorname{erf}\left(\alpha e^{-c}\right) \\
\int_{0}^{\infty} \operatorname{erf} i(a x) e^{-b^{2} x^{2}} \frac{d x}{x}=\sin ^{-1}\left(\frac{a}{b}\right), b \geqslant a
\end{gathered}
$$




$$
\begin{aligned}
& \int_{a}^{\infty} e^{x^{2}}\left(x^{2}-a^{2}\right)^{-1 / 2} \operatorname{erf} c(x) d x=\frac{1}{2} e^{a^{2} / 2} K_{0}\left(a^{2} / 2\right) \\
& \int_{0}^{\infty} \operatorname{erf} i(a x) e^{-a^{2} x^{2}} \frac{x}{x^{2}+b^{2}} d x=\frac{\pi}{2} \operatorname{erf} c(a b) e^{a^{2} b^{2}}
\end{aligned}
$$

\subsection{Definite Integrals from Laplace Transforms Involving Enf $(\sqrt{\mathrm{ax}})$}

Corrections

(12) Replace erf $(\sqrt{a x})$ in integrand by erfc $(\sqrt{a x})$ and remove the condition $b>a$.

(13) First term inside square brackets in integrand should read $(2 a x+1) x e^{a x}$ erf $c(\sqrt{a x})$ instead of $\left(2 a^{1 / 2} x^{1 / 2}+1\right) x e^{a x}$ erf $(\sqrt{a x})$ and remove the condition $b>a$.

(14) Last term inside square brackets in integrand should read

$$
-2\left(a x^{3} / \pi\right)^{1 / 2}(2 a x+5) \text { instead of }-2\left(a^{3} x^{5} / \pi\right)^{1 / 2}(2 a x+5) .
$$

(15) Replace erfc $(\sqrt{c x})$ in integrand by erf $(\sqrt{c x})$ and add the condition $b>c$.

(16) First term inside square brackets in integrand should read

$$
a^{1 / 2} e^{c x} \operatorname{erf}(\sqrt{c x}) \text { instead of } a^{1 / 2} e^{c x} \operatorname{erf}(\sqrt{b x}) .
$$

(18) First term inside brackets in integrand should read

$$
a(x / \pi)^{1 / 2} e^{-a^{2} /(4 x)}
$$

\section{Additions}

$$
\begin{gathered}
\int_{0}^{\infty} e^{-x} \frac{\operatorname{erf}(\sqrt{x})}{\left(1+e^{-x}\right)^{2}} d x=1+(\sqrt{2}-1) \zeta\left(\frac{1}{2}\right) \\
\int_{0}^{\infty} e^{-x} \operatorname{erf}(\sqrt{a x})\left[\frac{1-e^{-n x}}{1-e^{-x}}\right] \frac{d x}{\sqrt{x}}=\frac{2}{\sqrt{\pi}} \sum_{k=1}^{n} \frac{1}{\sqrt{k}} \tan ^{-1}[\sqrt{(a / k)}] \\
\int_{0}^{\infty} e^{-b x} \operatorname{erf}(\sqrt{a x}) e^{-n c x}[\sin h c x]^{n} \frac{d x}{\sqrt{x}} \\
=\frac{2}{\sqrt{\pi}} \frac{1}{2^{n}} \sum_{k=0}^{n}(-1)^{k}\left(\begin{array}{c}
n \\
k
\end{array}\right)(b+2 c k)^{-1 / 2} \tan ^{-1}\left(\frac{a}{b+2 c k}\right)^{1 / 2}
\end{gathered}
$$

\subsection{Combination of Error Function with Trigonometric Functions}

\section{Corrections}

(10) For the form given $a<b$.

For $a>b$ :

$$
\int_{0}^{\infty} \operatorname{erf}(a x) \sin b^{2} x^{2} d x=\frac{1}{4 b \sqrt{(2 \pi)}}\left[\ln \left(\frac{a^{2}+b^{2}+a b \sqrt{2}}{a^{2}+b^{2}-a b \sqrt{2}}\right)+2 \tan ^{-1}\left(\frac{a b \sqrt{2}}{b^{2}-a^{2}}\right)+2 \pi\right]
$$


(11) Change the condition on $p$ to $\mathscr{R}(p)>-2$.

(12) Change the condition on $p$ to $\mathscr{R}(p)>-1$.

$$
\begin{gathered}
\int_{0}^{\infty} \operatorname{erf}(a x) \sin b x \frac{d x}{x^{2}}=\frac{b}{2} E_{1}\left(\frac{b^{2}}{4 a^{2}}\right)+a \sqrt{\pi} \operatorname{erf}\left(\frac{b}{2 a}\right) . \\
\int_{0}^{\infty} \operatorname{erf}\left(\sqrt{\frac{a}{x}}\right) \sin b x d x=\frac{1}{b}\left[1-e^{-A} \cos A\right], \quad A=(2 a b)^{1 / 2} \\
\int_{0}^{\infty} \operatorname{erf}\left(\sqrt{\frac{a}{x}}\right) \cos b x d x=\frac{1}{b} e^{-A} \sin A, \quad A=(2 a b)^{1 / 2} \\
\int_{0}^{\infty} \operatorname{erf} c(a x) x \sin x \sin h x d x=\frac{1}{2}\left[\frac{1}{a^{2}} \sin \left(\frac{1}{2 a^{2}}\right)+\cos \left(\frac{1}{2 a^{2}}\right)-1\right]
\end{gathered}
$$

\section{Additions}

$$
2 i\left(b^{2}+c^{2}\right) \int \operatorname{erf}(a x) e^{-b x} \sin c x d x=(b+i c) A_{-}-(b-i c) A_{+},
$$

where

$$
\begin{gathered}
A_{ \pm}=\exp \left[\left(\frac{b \pm i c}{2 a}\right)^{2}\right] \operatorname{erf}\left(a x+\frac{b \pm i c}{2 a}\right)-\exp [-(b \pm i c) x] \operatorname{erf}(a x) \\
\int \tan [\operatorname{erf}(a x)] e^{-a^{2} x^{2}} d x=-\frac{\sqrt{\pi}}{2 a} \ln \{\cos [\operatorname{erf}(a x)]\}
\end{gathered}
$$

$$
\begin{aligned}
& \int_{0}^{\infty} \operatorname{erf}(a x) e^{-b^{2} x^{2}} \sin c x d x=\frac{\sqrt{\pi}}{2 b} e^{-c^{2} /\left(4 b^{2}\right)} \quad \operatorname{erf} i\left[\frac{a c}{2 b\left(a^{2}+b^{2}\right)^{1 / 2}}\right] \\
& \int_{0}^{\pi} \operatorname{erf}(a \sin x) \cos (2 n+1) x d x=\int_{0}^{\pi} \operatorname{erf}(a \sin x) \sin 2 n x d x=0
\end{aligned}
$$

$$
\int_{0}^{2 \pi} \operatorname{erf}(a \sin x+b \cos x) d x=0
$$

$$
\begin{gathered}
\int_{0}^{2 \pi} \operatorname{erf}\left(a e^{i x}\right) \sin n x d x=\left\{\begin{array}{cc}
0 & n=2 k \\
2 \sqrt{\pi} \frac{(-1)^{k}}{k !} \frac{a^{2 k+1}}{2 k+1} i & n=2 k+1
\end{array}\right. \\
\int_{0}^{2 \pi} \operatorname{erf}\left(a e^{i x}\right) \cos n x d x=\left\{\begin{array}{cc}
0 & n=2 k \\
2 \sqrt{\pi} \frac{(-1)^{k}}{k !} \frac{a^{2 k+1}}{2 k+1} & n=2 k+1
\end{array}\right.
\end{gathered}
$$

$$
\begin{aligned}
& \int_{0}^{\pi / 2} \operatorname{erf}(a \tan x) \sin 2 x d x=\sqrt{\pi} a e^{a^{2}} \operatorname{erf} c(a) \\
& \int_{0}^{\pi / 2} \operatorname{erf} c(a \tan x) \cos 2 x d x=\pi^{-1 / 2} a e^{a^{2}} E_{1}\left(a^{2}\right)
\end{aligned}
$$


(40) $\int_{0}^{1} \operatorname{erf}(a x) \cos \left[(2 n+1) \cos ^{-1} x\right]\left(1-x^{2}\right)^{-1 / 2} d x$

$$
=(-1)^{n} \frac{\Gamma\left(n+\frac{1}{2}\right)}{(2 n+1) !} \frac{e^{-a^{2} / 2}}{2 a} M_{-1 / 2, n+1 / 2}\left(a^{2}\right)
$$

(41) $a \int_{0}^{\infty} \operatorname{erf} c\left[b^{1 / 2}\left\{\left(x^{2}+c^{2}\right)^{1 / 2}-c\right\}^{1 / 2}\right] \sin a x d x=1-\frac{1}{\Delta}\left[\frac{1}{2} b(b+\Delta)\right]^{1 / 2} e^{-c(\Delta-b)}, \Delta=\left(a^{2}+b^{2}\right)^{1 / 2}$

$$
\int_{0}^{\infty}\left[\operatorname{erf}\left(\tan ^{-1} a x\right)-\operatorname{erf}\left(\tan ^{-1} b x\right)\right] \frac{d x}{x}=\ln \left(\frac{a}{b}\right) \operatorname{erf}\left(\frac{\pi}{2}\right)
$$

\subsection{Combination of Error Function With Logarithms and Powers}

\section{Corrections}

$$
\text { Replace } \frac{1}{2} z^{2} \text { erf }(a z) \ln z \text { on the R.H.S. by } \frac{1}{2} z^{2} \text { erf } c(a z) \ln z \text {. }
$$

\section{Additions}

(10)

$$
\begin{gathered}
\int_{0}^{\infty} \operatorname{erf}(a x) \ln (2 \pm 2 \cos x) \frac{x}{x^{2}+c^{2}} d x=-\pi \ln \left(1 \pm e^{-c}\right) \operatorname{erf} i(a c) \\
\int_{0}^{1} \operatorname{erf}(a \ln x) \frac{x^{b-1}}{1+x^{c}} d x=\frac{1}{c} \beta\left(\frac{b}{c}\right) \operatorname{erf}\left(\frac{a}{2 c}\right) \\
\int_{0}^{\infty} \operatorname{erf}(a \ln x) \frac{x^{b-1}}{1+x^{c}} d x=\frac{1}{c}\left[\beta\left(\frac{b}{c}\right)-\beta\left(1-\frac{b}{c}\right)\right] \operatorname{erf}\left(\frac{a}{2 c}\right), \quad c>b \\
\int_{0}^{\infty} \ln \left[\frac{c+d \operatorname{erf}(a x)}{c+d \operatorname{erf}(b x)}\right] \frac{d x}{x}=\ln \left(\frac{a}{b}\right) \ln \left(\frac{c+d}{c}\right) \\
\operatorname{erf~}(b \sin 2 x) \ln (a \tan x) d x=\frac{1}{2}(\ln a) \operatorname{erf}(2 b) \frac{\left[\Gamma\left(\frac{1}{2} a\right)\right]^{2}}{\Gamma(a)} \\
\int_{0}^{\pi / 2} \operatorname{erf}(a \ln \tan x) d x=0 \\
\int_{0}^{\infty} \operatorname{erf}\left(x^{p}+x^{-p}\right) \ln x \frac{d x}{1+x^{2}}=0 \\
\int_{0}^{\infty} \operatorname{erf}\left(\frac{a x}{x^{2}+b^{2}}\right) \ln \left(\frac{x}{b}\right) \frac{d x}{x}=0 \\
\left.x^{p}+b^{2 p}\right) \ln \left(\frac{x}{b}\right) \frac{d x}{x^{2}+b^{2}}=0 \\
\left.\int^{p}+x^{-p}\right) \ln x \frac{d x}{x}=0
\end{gathered}
$$




$$
\int_{0}^{\infty} \operatorname{erf}(a \ln x) \frac{d x}{1+b x+x^{2}}=0,|b|<2
$$

(22) $2 a^{2} \int_{0}^{\infty} x \ln x \operatorname{erf} c(x) e^{-a^{2} x^{2}} d x$

$$
=-\frac{1}{2} \gamma-\ln \left[1+\left(1+a^{2}\right)^{1 / 2}\right]+\frac{1}{2}\left(a^{2}+1\right)^{-1 / 2}\left[\gamma+2 \ln 2+\ln \left(1+a^{2}\right)\right]
$$

\subsection{Combination of Two Error Functions}

\section{Corrections}

(3) Last term on R.H.S. should read $+\frac{2 b}{\sqrt{\pi}} E_{1}(2 a b)$

(4) Last term on R.H.S. should read $-\frac{2 b}{\sqrt{\pi}} E_{1}(2 a b)$

$$
\int_{0}^{1} \operatorname{erf}(x) \operatorname{erf}\left[\left(1-x^{2}\right)^{1 / 2}\right] x d x=(2 e)^{-1}
$$

(8) Remove the entire expression and replace by

$$
12 b^{3} \sqrt{\pi} \int_{0}^{\infty} x^{2} \operatorname{erf} c(b x) \operatorname{erf} c\left(\frac{a}{2 x}\right) d x=e^{-a b}\left(4+a b-a^{2} b^{2}\right)+a^{3} b^{3} E_{1}(a b) .
$$

(9) Add the condition $c>a$.

\section{Additions}

(10) $\int \operatorname{erf}(a x) \operatorname{erf}(b x) d x=x \operatorname{erf}(a x) \operatorname{erf}(b x)-\frac{1}{a b \sqrt{\pi}}\left(a^{2}+b^{2}\right)^{1 / 2} \operatorname{erf}\left[x \sqrt{\left(a^{2}+b^{2}\right)}\right]$

$$
+\frac{1}{a \sqrt{\pi}} \operatorname{erf}(b x) e^{-a^{2} x^{2}}+\frac{1}{b \sqrt{\pi}} \operatorname{erf}(a x) e^{-b^{2} x^{2}}
$$

(11) $(2 n+1) \int x^{2 n} \operatorname{erf}(a x) \operatorname{erf}(b x) d x$

$$
\begin{gathered}
=x^{2 n+1} \operatorname{erf}(a x) \operatorname{erf}(b x)-\frac{2 b}{\sqrt{\pi}} \int x^{2 n+1} \operatorname{erf}(a x) e^{-b^{2} x^{2}} d x-\frac{2 a}{\sqrt{\pi}} \int x^{2 n+1} \operatorname{erf}(b x) e^{-a^{2} x^{2}} d x \\
\int x^{2 n} \operatorname{erf}^{2}(a x) d x=\frac{x^{2 n+1}}{2 n+1} \operatorname{erf}^{2}(a x)-\frac{4}{2 n+1} \frac{n !}{\sqrt{\pi} a^{2 n+1}} A,
\end{gathered}
$$


where

$A=\frac{1}{2 \sqrt{2}} \operatorname{erf}\left(\begin{array}{ll}a x & \sqrt{2}\end{array}\right) \sum_{k=0}^{n}\left(\begin{array}{c}2 k \\ k\end{array}\right) 8^{-k}-\frac{1}{2} \operatorname{erf}(a x) e^{-a^{2} x^{2}} e_{n}\left(a^{2} x^{2}\right)$

$$
-\frac{a x}{\sqrt{\pi}} e^{-2 a^{2} x^{2}} \sum_{k=0}^{n}\left(\begin{array}{c}
2 k \\
k
\end{array}\right) 8^{-k} \sum_{l=0}^{k-1} \frac{l !}{(2 l+1) !}\left(8 a^{2} x^{2}\right)^{l}
$$

(13)

$$
\int x \operatorname{erf}^{2}(a x) d x=\left(\frac{x^{2}}{2}-\frac{1}{4 a^{2}}\right) \operatorname{erf}^{2}(a x)+\frac{x}{a \sqrt{\pi}} \operatorname{erf}(a x) e^{-a^{2} x^{2}}+\frac{1}{2 \pi a^{2}} e^{-2 a^{2} x^{2}}
$$

$$
\int x^{2 n+1} \operatorname{erf}^{2}(a x) d x=\left[x^{2 n+2}-\frac{2}{n !} \frac{(2 n+1) !}{(2 a)^{2 n+2}}\right] \frac{\operatorname{erf}^{2}(a x)}{2 n+2}+\frac{2}{(2 a)^{2 n+2}} \frac{(2 n+1) !}{(n+1) !} B,
$$

where

$$
B=\frac{2 a x}{\sqrt{\pi}} \operatorname{erf}(a x) e^{-a^{2} x^{2}} \sum_{k=0}^{n} \frac{k !}{(2 k+1) !}\left(4 a^{2} x^{2}\right)^{k}+\frac{e^{-2 a^{2} x^{2}}}{\pi} \sum_{k=0}^{n} \frac{2^{k} k ! k !}{(2 k+1) !} e_{k}\left(2 a^{2} x^{2}\right)
$$

$$
\int_{0}^{\infty} \operatorname{erf}(a x) \operatorname{erf}(b x) \frac{d x}{x^{2}}=\frac{2}{\sqrt{\pi}}\left[a \ln \left(\frac{b+c}{a}\right)+b \ln \left(\frac{a+c}{b}\right)\right], c=\left(a^{2}+b^{2}\right)^{1 / 2}
$$

$$
\begin{aligned}
\int_{0}^{\infty} x^{p-1} \operatorname{erf} c(a x) \operatorname{erf}(b x) d x= & \frac{2 b}{a^{p+1}} \frac{\Gamma(1+p / 2)}{\pi(p+1)}{ }_{3} F_{2}\left(\frac{1}{2}, \frac{p+1}{2}, \frac{p+2}{2} ; \frac{3}{2}, \frac{p+3}{2} ;-\frac{b^{2}}{a^{2}}\right), \\
& a>b, \quad p>-1
\end{aligned}
$$

(17) $\int_{0}^{\infty} \operatorname{erf}(a x) \operatorname{erf}(b x) e^{-c^{2} x^{2}} \frac{d x}{x^{2}}$

$$
\begin{gathered}
=\frac{2}{\sqrt{\pi}}\left\{a \ln \left[\frac{b+\Delta}{\left(a^{2}+c^{2}\right)^{1 / 2}}\right]+b \ln \left[\frac{a+\Delta}{\left(b^{2}+c^{2}\right)^{1 / 2}}\right]-c \tan ^{-1}\left(\frac{a b}{c \Delta}\right)\right\}, \\
\Delta=\left(a^{2}+b^{2}+c^{2}\right)^{1 / 2}
\end{gathered}
$$

(18)

$$
\int_{0}^{\infty} \operatorname{erf}(a x) \operatorname{erf}(b x) e^{-c^{2} x^{2}} d x=\frac{1}{c \sqrt{\pi}} \tan ^{-1}\left(\frac{a b}{c \Delta}\right), \Delta=\left(a^{2}+b^{2}+c^{2}\right)^{1 / 2}
$$

$$
\begin{aligned}
& \int_{0}^{\infty} \operatorname{erf}(a x) \operatorname{erf}(b x) e^{-c^{2} x^{2}} x d x=\frac{1}{c^{2} \pi}\left[\frac{b}{\Delta_{2}} \tan ^{-1} \frac{a}{\Delta_{2}}+\frac{a}{\Delta_{1}} \tan ^{-1} \frac{b}{\Delta_{1}}\right] \\
& \Delta_{1}=\left(a^{2}+c^{2}\right)^{1 / 2}, \Delta_{2}=\left(b^{2}+c^{2}\right)^{1 / 2}
\end{aligned}
$$

$$
\begin{gathered}
\int_{0}^{\infty} x e^{x^{2}} \ln x \operatorname{erf} c^{2}(x) d x=\frac{2}{\pi}\left[G-1-\frac{\gamma}{2}\left(1-\frac{\pi}{4}\right)\right] \\
\int_{0}^{\infty}\left[1-\operatorname{erf}^{3}(x)\right] d x=\frac{6}{\pi}\left(\frac{2}{\pi}\right)^{1 / 2} \tan ^{-1}\left(2^{-1 / 2}\right) \\
\int_{0}^{\infty}\left[1-\operatorname{erf}^{4}(x)\right] d x=\frac{12}{\pi}\left(\frac{2}{\pi}\right)^{1 / 2} \tan ^{-1}\left(8^{-1 / 2}\right)
\end{gathered}
$$




\subsection{Combination of Error Function with Bessel Functions}

\section{Corrections}

(12) Replace $\Gamma\left(-p-1, \frac{b^{2}}{4 a^{2}}\right)$ on the R.H.S. by $\Gamma\left(-p-\frac{1}{2}, \frac{b^{2}}{4 a^{2}}\right)$.

(13) Replace $W_{-p / 2, p / 2}\left(\frac{b^{2}}{8 a^{2}}\right)$ by $W_{-p / 2, p / 2}\left(\frac{b^{2}}{4 a^{2}}\right)$;

replace $b^{p+1 / 2} / a^{3 / 2 p+1}$ by $b^{p} / a^{2 p+1}$;

change the condition $-1<p<\frac{3}{2}$ to $-\frac{1}{2}<p<\frac{3}{2}$.

(19) Multiply the R.H.S. by $\frac{2}{\sqrt{\pi}}$;

change the condition on $p$ to $-\frac{1}{2}<p<0$.

(20) Multiply the R.H.S. by $\frac{2}{\sqrt{\pi}}$

(21) Replace the condition $\lambda+\frac{1}{2} p>0$ by $\lambda+\frac{1}{2} p>-\frac{1}{2}$

\section{Additions}

$$
\begin{aligned}
& \int_{0}^{\infty} J_{1}[a \text { erf }(x)] e^{-x^{2}} d x=\frac{\sqrt{\pi}}{2 a}\left[1-J_{0}(a)\right] \\
& \int_{0}^{\infty} J_{0}\left[a \sqrt{\left\{1-\operatorname{erf}^{2}(x)\right\}}\right] e^{-x^{2}} d x=\frac{\sqrt{\pi}}{2 a} \sin a
\end{aligned}
$$$$
\int_{0}^{\infty} K_{0}(a x) \operatorname{erf}(b x) x d x=\frac{1}{8 b^{2}} e^{c}\left[K_{1}(c)-K_{0}(c)\right], c=a^{2} /\left(8 b^{2}\right)
$$

$$
\int_{0}^{\infty} e^{-x^{2}} I_{0}\left(x^{2}\right) \text { erf } c(a x) d x=(8 \pi)^{-1 / 2} \ln \left[1+\frac{2}{a^{2}}+\frac{2}{a^{2}}\left(1+a^{2}\right)^{1 / 2}\right]
$$

(26) $\int_{0}^{\infty} x^{2 p-2} e^{-x^{2}} K_{\nu}\left(x^{2}\right) \operatorname{erf}(x) d x=\frac{\Gamma(p+\nu) \Gamma(p-\nu)}{2^{p} \Gamma\left(p+\frac{1}{2}\right)}{ }_{3} F_{2}\left(p+\nu, p-\nu, \frac{1}{2} ; p+\frac{1}{2}, \frac{3}{2} ;-\frac{1}{2}\right)$,

$$
p>|\nu|
$$

(27) $\int_{0}^{\infty} x^{2 \lambda} J_{2 v}\left(\frac{a}{x}\right) \operatorname{erf}(b x) d x$

$$
\begin{gathered}
=\left(\frac{a}{2}\right)^{2 \nu} \frac{b^{2 \nu-2 \lambda-1} \Gamma(\lambda-\nu+1)}{\sqrt{\pi}(2 \nu-2 \lambda-1) \Gamma(2 \nu+1)}{ }_{1} F_{3}\left(\nu-\lambda-\frac{1}{2} ; 2 \nu+1, \nu-\lambda, \nu-\lambda+\frac{1}{2} ; \frac{a^{2} b^{2}}{4}\right) \\
+\left(\frac{a}{2}\right)^{2 \lambda+2} \frac{b \Gamma(\nu-\lambda-1)}{\sqrt{\pi} \Gamma(\nu+\lambda+2)}{ }_{1} F_{3}\left(\frac{1}{2} ; \frac{3}{2}, \lambda-\nu+2, \lambda+\nu+2 ; \frac{a^{2} b^{2}}{4}\right), \\
\nu>\lambda>-\frac{5}{4}
\end{gathered}
$$




\subsection{Combination of Error Function with Other Special Functions}

\section{Corrections}

(3) Replace $\operatorname{si}(2 p x)$ in integrand by $\frac{1}{a} \operatorname{si}\left(\frac{x}{a}\right)$.

(5) Replace $e^{a^{2} / 4}$ on R.H.S. by $e^{a^{2 / 2}}$;

change second index of $W$ from $(1+2 \mu) / 4$ to $(1+2 \nu) / 4$;

change argument of $W$ from $a^{2}$ to $a^{2} / 2$;

change the condition $\mu<\nu$ to $\mu>\nu$.

(6) Add the condition $\nu>-\frac{1}{2}$.

(7) Add the condition $\nu>-\frac{1}{2}$.

(9) Change the R.H.S. to

$$
\frac{1}{\sqrt{\pi}} \frac{\Gamma(b)}{p^{2 b-1}}(2 a-2 b+1)^{-1}{ }_{2} F_{1}\left(a, a-b+\frac{1}{2} ; a-b+\frac{3}{2} ;-1\right), \quad a+\frac{1}{2}>b
$$

(10) Change the R.H.S. to

$$
\frac{1}{\sqrt{\pi}} \frac{\Gamma(b)}{p^{2 b-1}}(p / q)^{2 a}(2 a-2 b+1)^{-1}{ }_{2} F_{1}\left(a, a-b+\frac{1}{2} ; a-b+\frac{3}{2} ;-\frac{p^{2}}{q^{2}}\right), \quad a+\frac{1}{2}>b ; q^{2} \geqslant p^{2}
$$

(11) Change the R.H.S. to

$\frac{1}{\sqrt{\pi}} \frac{p}{q^{\nu+2}} \Gamma\left(1+\frac{\nu}{2}\right) \frac{\Gamma(b) \Gamma\left(a-1-\frac{1}{2} \nu\right)}{\Gamma(a) \Gamma\left(b-1-\frac{1}{2} \nu\right)}{ }_{3} F_{2}\left(\frac{1}{2}, 1+\frac{\nu}{2}, 2-b+\frac{\nu}{2} ; \frac{3}{2}, 2-a+\frac{\nu}{2} ;-\frac{p^{2}}{q^{2}}\right), \quad \nu>-2 ; q^{2} \geqslant p^{2}$

(16) Change the condition on $p$ to $p>0$.

(18) Integrand should contain erfc $\left(\sqrt{\frac{a}{2}} x\right)$ instead of erfc $\left(\frac{a}{\sqrt{2}} x\right)$;

coefficient on R.H.S. should read

$$
2^{\nu / 2+1} a^{-3 / 2} /\left[\left(1+\frac{\nu}{2}\right) \Gamma\left(1-\frac{\nu}{2}\right)\right] \text { instead of } 2^{\nu / 2+2} a^{-3 / 2} /(\nu \pi)
$$

(20) Replace $p^{\nu+1 / 2}$ on the R.H.S. by $a^{\nu+1 / 2}$

(21) First term inside ${ }_{3} F_{2}$ expression should read

$$
-\lambda+\mu+\frac{1}{2} \text { instead of } \lambda+\mu+\frac{1}{2}
$$

argument of ${ }_{3} F_{2}$ should read $+a$ instead of $-a$;

add the condition $a \leqslant 1 ; a=1, \lambda>\frac{1}{2} p$. 


\section{Additions}

$(22)$

$$
\int_{0}^{1} T_{2 n+1}(x) \operatorname{erf}(a x)\left(1-x^{2}\right)^{-1 / 2} d x=(-1)^{n} \frac{\Gamma\left(n+\frac{1}{2}\right)}{2 a(2 n+1) !} e^{-a^{2 / 2}} M_{-1 / 2, n+1 / 2}\left(a^{2}\right)
$$

$$
\int_{-\infty}^{\infty}\left\{T_{n}[\operatorname{erf}(x)]\right\}^{2} e^{-x^{2}} d x=\sqrt{\pi}\left(\frac{2 n^{2}-1}{4 n^{2}-1}\right)
$$

(24) $\int_{0}^{1} P_{n}\left(1-2 x^{2}\right) \operatorname{erf}(a x) d x$

$$
=\left(\frac{a}{2}\right)^{2 n+1} \frac{1}{(2 n+1) \Gamma\left(n+\frac{3}{2}\right)}{ }_{2} F_{2}\left(n+\frac{1}{2}, n+1 ; n+\frac{3}{2}, 2 n+2 ;-a^{2}\right)
$$

(25)

$$
\int_{0}^{1} P_{2 n+1}(x) \text { erf }(a x) \frac{d x}{\sqrt{x}}=(-1)^{n+1} \frac{1}{2 a \sqrt{(a \pi)}} \frac{\Gamma\left(n+\frac{1}{2}\right)}{\Gamma\left(2 n+\frac{5}{2}\right)} e^{-a^{2 / 2}} M_{-3 / 4}, n+3 / 4\left(a^{2}\right)
$$

(26) $\int_{0}^{1}\left(1-x^{2}\right) P_{2 n+1}^{(p, p)}(x) \operatorname{erf}(a x) d x$

$$
=\frac{(-1)^{n}}{2 \sqrt{\pi}} \frac{\Gamma\left(n+\frac{1}{2}\right)}{(2 n+1) !} \frac{\Gamma(2 n+p+2)}{\Gamma\left(2 n+p+\frac{5}{2}\right)} \frac{e^{-i^{2 / 2}}}{a^{p+3 / 2}} M_{-p / 2-3 / 4, n+p / 2+3 / 4} \quad\left(a^{2}\right), p>-1
$$

$(27)$

$\int_{0}^{\infty} x^{\lambda-1}\left(1-x^{2}\right)^{-\mu / 2} p_{\nu}^{\mu}(x) \operatorname{erf}(a x) d x$

$=\frac{2^{\mu-\lambda} a \Gamma(\lambda+1)}{\Gamma\left(1+\frac{1}{2} \lambda-\frac{1}{2} \mu-\frac{1}{2} \nu\right) \Gamma\left(\frac{3}{2}+\frac{1}{2} \lambda-\frac{1}{2} \mu+\frac{1}{2} \nu\right)}{ }_{3} F_{3}\left(\frac{1}{2}, \frac{\lambda+1}{2}, \frac{\lambda+2}{2} ; \frac{3}{2}, \frac{2+\lambda-\mu-\nu}{2}, \frac{3+\lambda-\mu-\nu}{2} ;-a^{2}\right)$,

$$
\lambda>-1 ; \mu<1
$$

(28)

$$
\int \operatorname{erf}(a x) H_{n}(a x) d x=[2 a(n+1)]^{-1}\left\{\operatorname{erf}(a x) H_{n+1}(a x)+\frac{2}{\sqrt{\pi}} H_{n}(a x) e^{-a^{2} x^{2}}\right\}
$$

$$
\int_{0}^{\infty} e^{-x^{2}} H_{2 n}(x) \operatorname{erf}(x) x d x=(-1)^{n+1} \frac{(2 n) !}{2^{n+3 / 2} n !} \frac{2 n+1}{2 n-1}
$$

$$
\int_{0}^{\infty} e^{-x^{2}} H_{n}(x) H_{n+2 m+1}(x) \operatorname{erf}(x) d x=(-1)^{m} \frac{(2 m+2 n+2) !}{(m+n+1) !} \frac{1}{2^{m+n+3 / 2}} \frac{1}{2 m+1}
$$

$$
\begin{array}{r}
\int_{0}^{\infty} x^{p-1} H_{2 n}(a x) \operatorname{erf} c(b x) d x=(-1)^{n} \frac{(2 n) ! \Gamma\left(\frac{1}{2}+\frac{1}{2} p\right)}{n ! p b^{p} \sqrt{\pi}}{ }_{3} F_{2}\left(-n, \frac{p}{2}, \frac{p+1}{2} ; \frac{1}{2}, \frac{p+2}{2} ; \frac{a^{2}}{b^{2}}\right) \\
p>0 ; b^{2}>a^{2}
\end{array}
$$


(33) $\int_{0}^{1}\left(1-x^{2}\right)^{p-1 / 2} C_{2 n+1}^{p}(x) \operatorname{erf}(a x) d x$

$$
\begin{gathered}
=\frac{(-1)^{n}}{2^{2 p}} \frac{a^{2 n+1}}{(2 n+1) !} \frac{\Gamma\left(n+\frac{1}{2}\right)}{\Gamma(p)} \frac{\Gamma(2 n+2 p+1)}{\Gamma(2 n+p+2)}{ }_{1} F_{1}\left(n+\frac{1}{2} ; 2 n+p+2 ;-a^{2}\right) \\
p>0
\end{gathered}
$$

$$
\int_{0}^{\infty} e^{-x^{2} / 4}\left[D_{2 p}(x)-D_{2 p}(-x)\right] \operatorname{erf}(x) d x=\left(\frac{4}{3}\right)^{p} \vee(2 / \pi) \sin p \pi \Gamma(p), p>0
$$

(36) $\int_{0}^{\infty} x^{2 p-1} e^{-x^{2} / 4} D_{2 q}(x) \operatorname{erf}\left(\frac{1}{2} x\right) d x$

$$
=2^{q-p-1 / 2} \frac{\Gamma(2 p+1)}{\Gamma(p-q+1)}{ }_{3} F_{2}\left(\frac{1}{2}, p+\frac{1}{2}, p+1 ; \frac{3}{2}, p-q+1 ;-\frac{1}{2}\right), p>-\frac{1}{2}
$$

$\int_{0}^{\infty} x^{2 n+1} e^{-x^{2} / 2} \Gamma{ }_{n}^{n+1 / 2}\left(x^{2} / 2\right) \operatorname{erf}(x) d x$

$$
=\frac{1}{\sqrt{\pi}}\left(\frac{2}{3}\right)^{n+1 / 2} \frac{2^{n}}{n !} \frac{\Gamma(2 n+3 / 2)}{\left(n+\frac{1}{2}\right)}{ }_{2} F_{1}\left(-n, n+\frac{1}{2} ; n+\frac{3}{2} ; \frac{2}{3}\right)
$$

(38) $\quad \int_{0}^{\infty} L_{n}^{\alpha}\left(a^{2} x^{2}\right) \operatorname{erf} c(b x) x^{p-1} d x$

$$
=\left(\begin{array}{c}
\alpha+n \\
n
\end{array}\right) \frac{\Gamma\left(\frac{1}{2}+\frac{1}{2} p\right)}{p b^{p} \sqrt{\pi}}{ }_{3} F_{2}\left(-n, \frac{p}{2}, \frac{p+1}{2} ; \alpha+1, \frac{p+2}{2} ; \frac{a^{2}}{b^{2}}\right), \alpha>-1 ; p>0 ; b^{2}>a^{2}
$$

$$
\int_{-\infty}^{\infty} \frac{\left[\operatorname{erf}\left(a e^{i \pi x}\right)-\operatorname{erf}\left(a e^{-i \pi x}\right)\right]}{\Gamma(\alpha+x) \Gamma(\beta-x) \sin \pi x} d x=i \frac{2^{\alpha+\beta-1}}{\Gamma(\alpha+\beta-1)} \operatorname{erf}(a), \alpha+\beta>1
$$

(40) $\quad \int_{0}^{\infty} x^{p-1} \operatorname{erf} c(b x) \gamma\left(\alpha, a^{2} x^{2}\right) d x$

$$
=\frac{a^{2 \alpha} \Gamma\left(\alpha+\frac{1}{2}+\frac{1}{2} p\right)}{\alpha \sqrt{\pi}(p+2 \alpha) b^{p+2 \alpha}{ }_{3} F_{2}}\left(\alpha, \alpha+\frac{1}{2} p, \alpha+\frac{1}{2}+\frac{1}{2} p ; \alpha+1, \alpha+1+\frac{1}{2} p ;-\frac{a^{2}}{b^{2}}\right), \quad p+2 \alpha>0 ; b^{2}>a^{2}
$$

(41) $\int_{3}^{\infty} \operatorname{erf}(a x)_{2} F_{1}\left(\alpha+1, \beta+1+3 / 2 ;-c^{2} x^{2}\right) x d x$

$$
=\frac{1}{4 \alpha \beta a^{2}}\left(\frac{a}{c}\right)^{\alpha+\beta+1} e^{a^{2} /\left(2 c^{2}\right)} W_{(1-\alpha-\beta) / 2,(\alpha-\beta) / 2}\left(\frac{a^{2}}{c^{2}}\right), \alpha>0 ; \beta>0
$$


(42)

$\int_{0}^{\infty} x^{4 p} e^{-a^{2} x^{2}}{ }_{1} F_{1}\left(\frac{1}{2}-2 p ; 2 p+1 ; a^{2} x^{2}\right) \operatorname{erf}(b x) d x$

$$
\begin{gathered}
=\frac{1}{2 a \sqrt{\pi}} \Gamma(2 p)\left[\frac{b^{2}}{a^{2}\left(a^{2}+b^{2}\right)}\right]^{2 p}{ }_{2} F_{1}\left(\frac{1}{2}-2 p, 2 p ; 2 p+1 ; \frac{b^{2}}{a^{2}+b^{2}}\right), \\
p>0
\end{gathered}
$$

(43) $\int_{0}^{\infty} e^{x^{2}} x^{2 \beta-2} F_{1}\left(\alpha ; \beta ;-\gamma^{2} x^{2}\right) \operatorname{erf} c(x) d x$

$$
\begin{aligned}
& =\frac{\Gamma(2 \beta-1) \Gamma(1+\alpha-\beta)}{2^{2 \beta-1} \Gamma\left(\frac{1}{2}+\alpha\right)}{ }_{2} F_{1}\left(\alpha, \beta-\frac{1}{2} ; \alpha+\frac{1}{2} ; 1-\nu^{2}\right), \\
\gamma^{2}<2 & ; 1+\alpha>\beta>\frac{1}{2}
\end{aligned}
$$

(44) $\int_{0}^{\infty} e^{-x^{2}} \Psi\left(\alpha, \beta ; \gamma^{2} x^{2}\right)$ erf $i(x) x d x$

$$
\begin{aligned}
& =\frac{1}{2 \gamma^{3}} \frac{\Gamma\left(\alpha-\frac{1}{2}\right) \Gamma(5 / 2-\beta)}{\Gamma(\alpha) \Gamma(\alpha-\beta+2)}{ }_{2} F_{1}\left(1, \frac{5}{2}-\beta ; \alpha-\beta+2 ; 1-\frac{1}{\gamma^{2}}\right), \\
& \alpha>\frac{1}{2} ; \gamma^{2}>\frac{1}{2} ; \beta<\frac{5}{2}
\end{aligned}
$$

(45) $\int_{0}^{\infty} e^{-x / 2} x^{p-1} W_{\alpha, \beta}(x) \operatorname{erf}\left(a^{1 / 2} x^{1 / 2}\right) d x$

$$
\begin{gathered}
=2 \sqrt{(a / \pi)} \frac{\Gamma(1+\beta+p) \Gamma(1-\beta+p)}{\Gamma(3 / 2-\alpha+p)}{ }_{3} F_{2}\left(\frac{1}{2}, 1+\beta+p, 1-\beta+p ; \frac{3}{2}, \frac{3}{2}-\alpha+p ;-a\right), \\
p+1>|\beta|
\end{gathered}
$$

\section{References}

[1] Ng, Edward W., and Geller, Murray, A Table of Integrals of the Error Functions, J. Res. Nat. Bur. Stand. (U.S.), 73B (Math. Sci.), No. 1, 1-20 (Jan.-Mar. 1969).
[2] Hadji, P. I., The Calculation of Some Integrals Containing a Probability Function, Moldavian Academy of Sciences SSR; Series of Physical, Tech. and Math. Sci. 2, 81 (1968).

(Paper 75B3\&4-355) 